\title{
Potential use of Embryonic antitumor modulator (EATM) for diabetes prevention and treatment
}

\section{Aghajanova Y. \\ Department of Endocrinology, Yerevan State Medical University, Yerevan, Armenia}

Introduction: Incidence of diabetes mellitus worldwide is on the rise, and better methods of prevention and treatment are needed. We evaluated the possible antidiabetic effect of
Embryonic antitumor modulator (EATM) created by L.Mkrtchyan, which reportedly exhibit anticancer, neuroprotective and immunomodulatory properties.
Materials and Methods: Rats with streptozotocinl (STZ)-induced diabetes ( $55 \mathrm{mg} / \mathrm{kg}$ once i.p.) were injected EATM ( $1 \mathrm{mg} / 200 \mathrm{~g}$ rat body mass once i.p.) in preventive mode and in
therapeutic mode. The activity of cortisol, prolactin, IL-4, $-6,-8$ in the serum, as well as releasing of NAPDH-oxidase in erythrocyte membranes and exosomes of blood serum were
studied. For morphological analysis pieces from the pancreas, liver, kidney and heart were stained with hematoxylin-eosin, aldehyde-fuchsin or Azur II-eosin.

Results: The activity of serum cortisol, prolactin (pic.1), IL-4, -6, -8 (pic.2,3,4), as well as releasing of NAPDH-oxidase in erythrocyte membranes and exosomes in blood largely normalized in treated compared to untreated STZ animals.

The content of proinflamatory cytokines in STZ-induced DM was sharply changed. As is shown in table 1, the level of IL-4 and -8 rised 12 times, whereas IL- 6 rised 25 times. Preliminary administration of EATM prevents such pronounced changes in studied interleukins content. Compared with DM content of the level of IL-4 and IL-8 was 2 times lower, what about IL-6 it's content was high, but remained lower than in DM group for 9\%. Although the level of the all interleukins in preventive group compared with control group, definitely were increased. All the mentioned changes were for certain.

These results coincide with the data of some investigators, who favors the primary role of cellular immunity and its humoral mediators in pathogenesis and following type I diabetes mellitus. It was shown, that circulating levels of IL-8 were elevated and were correlated with BMI in children with type I DM, hinting perhaps at adipose tissue as a site production. Elevated systemic IL-6 and TNF- $\alpha$ were limited to newly diagnosed cases suggesting activation of the inflammatory immune response system at early stages of the disease [2].

The morphological studies revealed (tab.2, pic.5), that there was a 4-fold decline in the number of pancreatic $\beta$-cells in STZ rats (Group 2) compared to control animals (Goup 1), while preventive injection of EATM (Group 3) animals showed significantly smaller decrease in $\beta$-cells, with levels being higher than in Group 2. In the EATM-treatment group (Group 4) the number of $\beta$-cells was the highest among the treated groups ( $\mathrm{p}>0.05)$, and was just 2 -fold lower than those of control Group 1 .

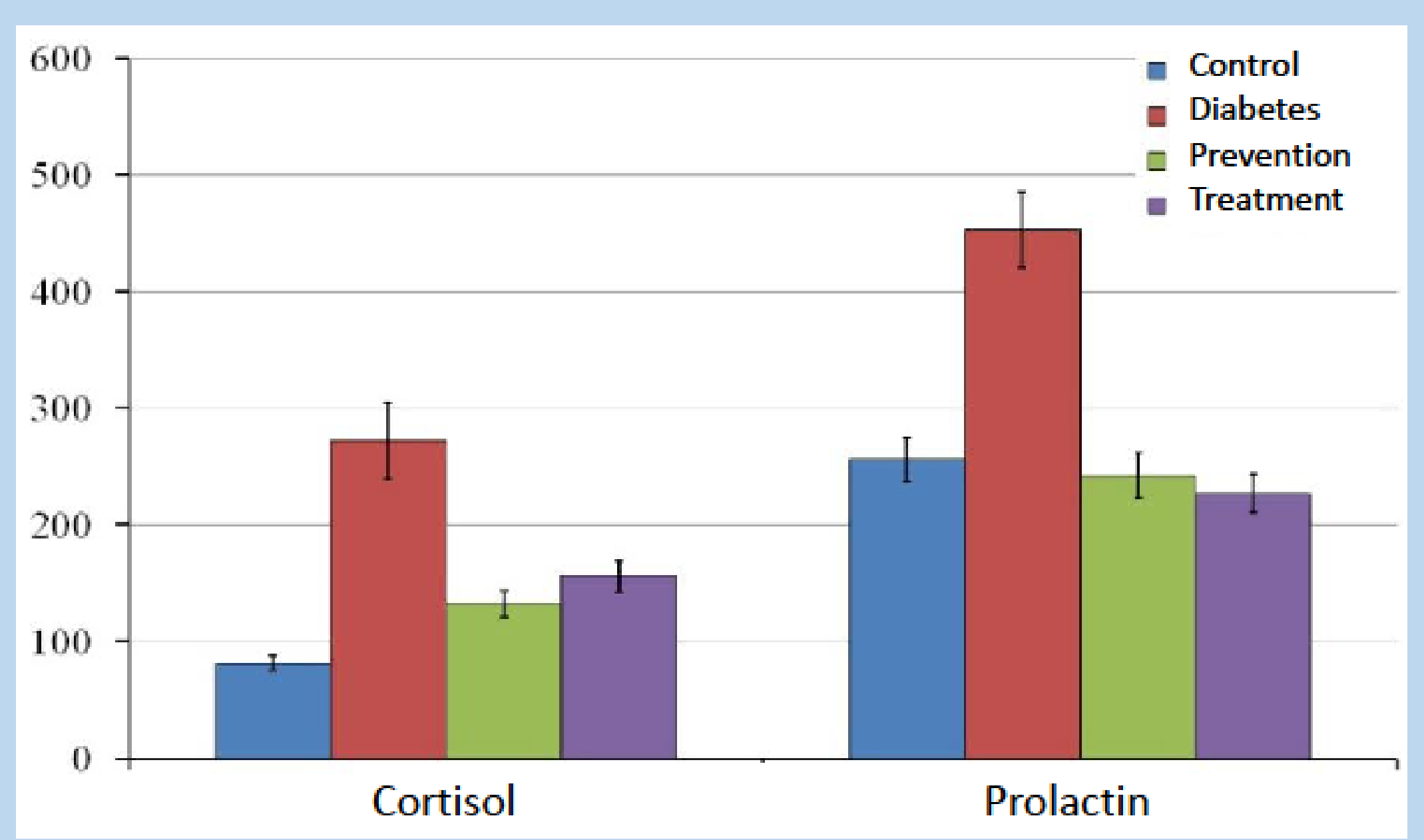

Pic.1. Effect of the EATM on the level of cortisol and prolactin in STZ-induced rats blood (\%)

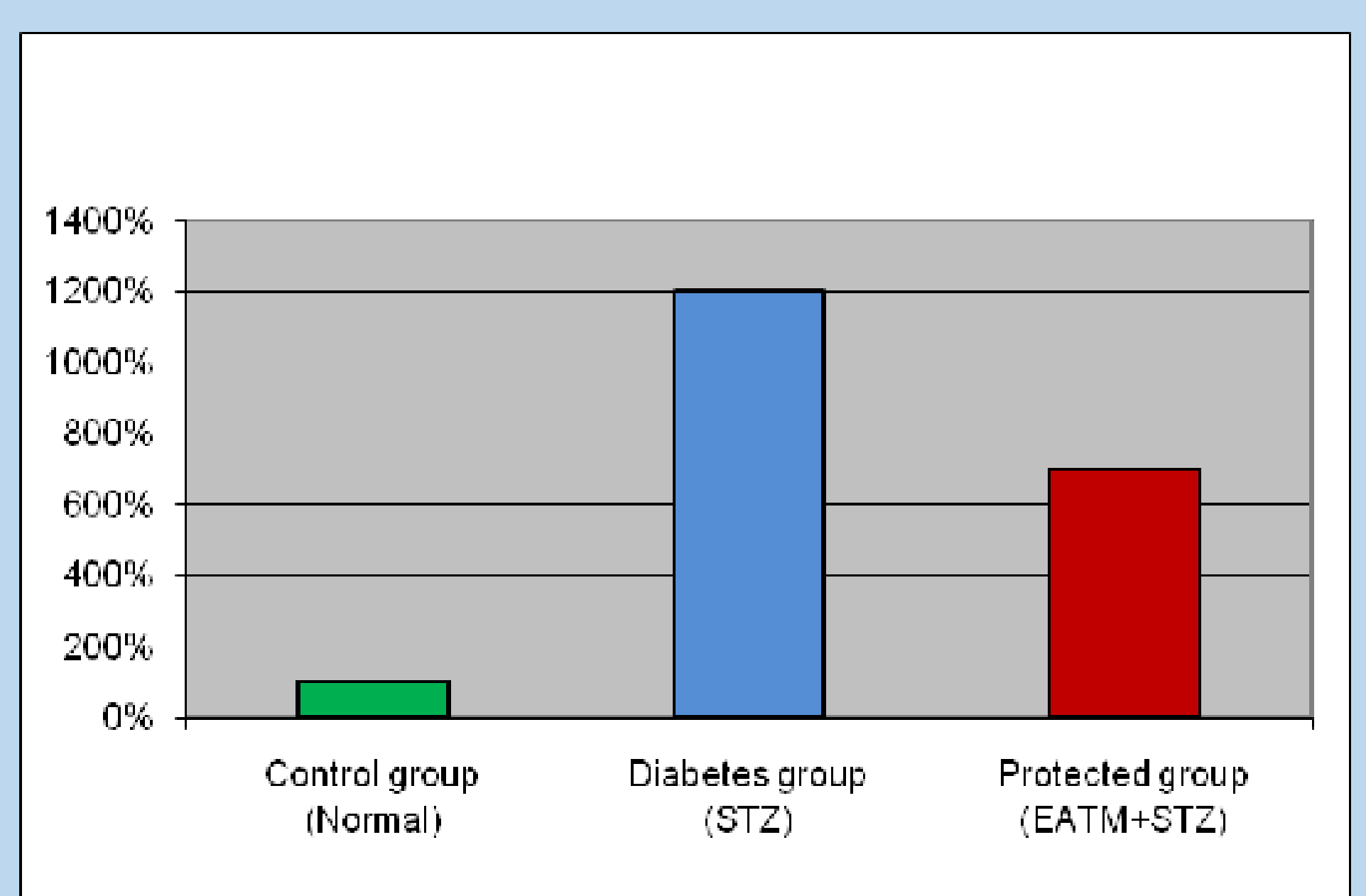

Pic.2. Interleukin 4 amount (in \%)

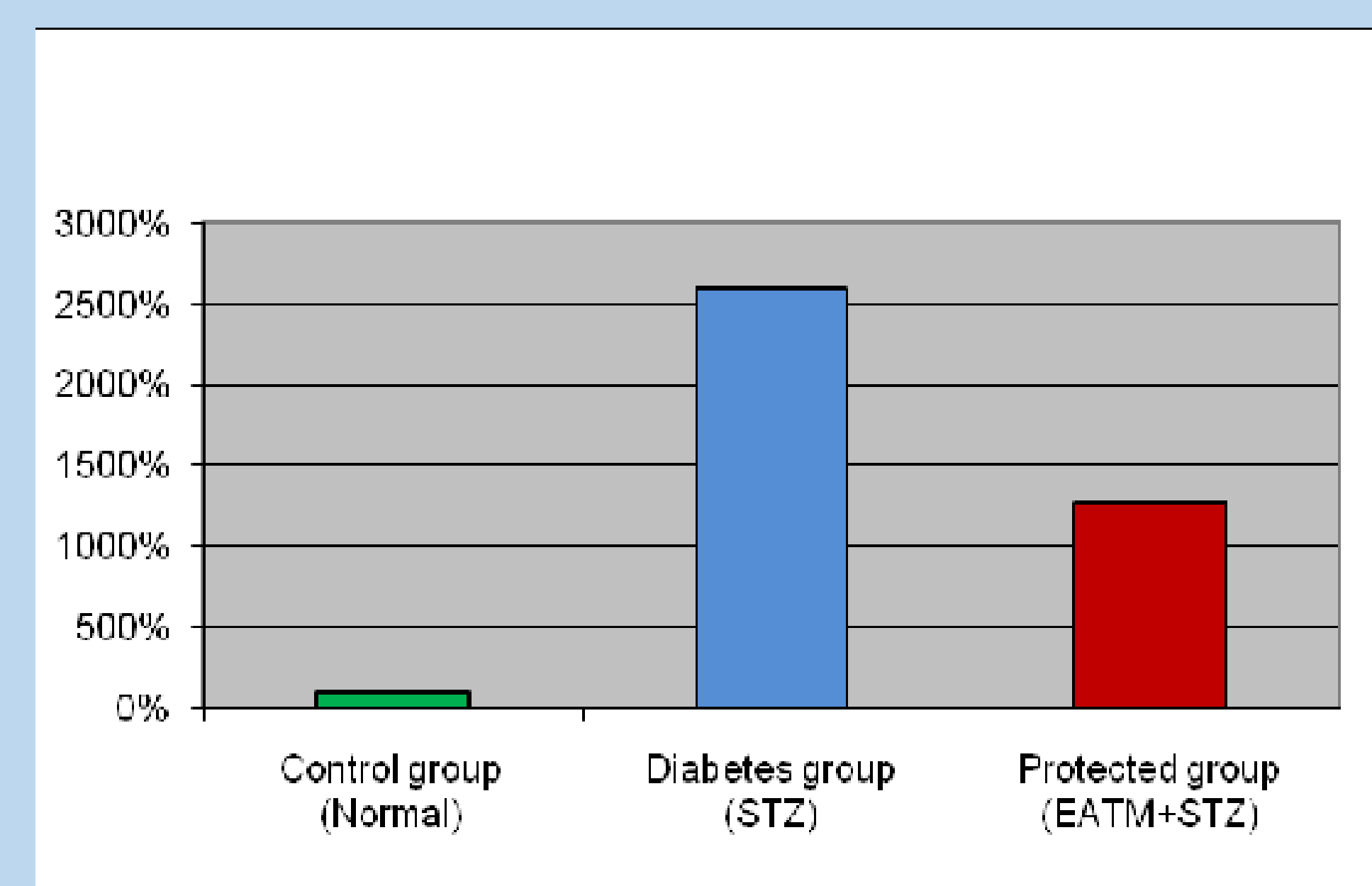

Pic.3. Interleukin 6 amount (in \%)

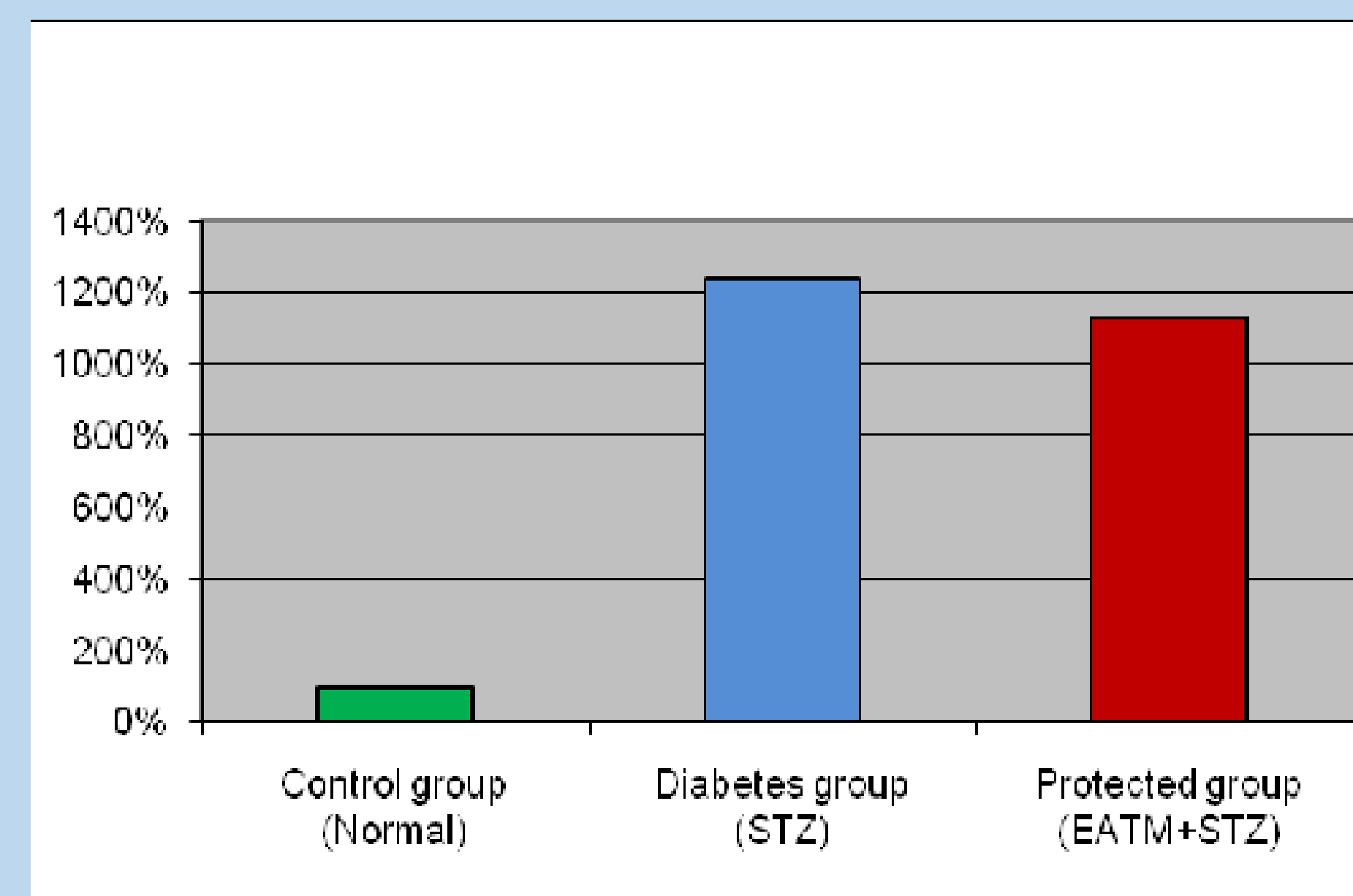

Pic.4. Interleukin 8 amount (in \%)

\begin{tabular}{|c|c|c|c|}
\hline$\underset{\mathrm{ns}}{\text { Interleuki }}$ & $\begin{array}{c}\text { Control } \\
(\mathrm{n}=5)\end{array}$ & $\begin{array}{l}\text { Diabetes (STZ) } \\
\qquad(\mathrm{n}=\mathbf{8})\end{array}$ & $\begin{array}{c}\text { Preventive(EATM+S } \\
\text { TZ) } \\
(\mathrm{n}=6)\end{array}$ \\
\hline IL-4 & $1,1 \pm 0,1$ & $\begin{array}{l}13,2 \pm 1,5 \\
p<0,001\end{array}$ & $\begin{array}{c}7,7 \pm 1,6 \\
\mathrm{p}_{1}<0,01 \mathrm{p}_{2}<0,02\end{array}$ \\
\hline IL-6 & $1,54 \pm 0,02$ & $\begin{array}{l}39,9 \pm 5,9 \\
\mathrm{p}<0,001\end{array}$ & $\begin{array}{c}19,6 \pm 4,0 \\
\mathrm{p}_{1}<0,001 \mathrm{p}_{2}<0,01\end{array}$ \\
\hline IL-8 & $1,45 \pm 0,075$ & $\begin{array}{c}18,0 \pm 0,63 \\
p<0,001\end{array}$ & $\begin{array}{c}16,4 \pm 0,74 \\
\mathrm{p}_{1}<0,001 \mathrm{p}_{2}<0,01\end{array}$ \\
\hline
\end{tabular}

Table 1. Effect of the EATM on the interleukins level in STZinduced diabetes mellitus rats blood $(\mathrm{pg} / \mathrm{ml})$

$p$ - control/diabet; $p_{1}$ - preventive/control; $p_{2}$ - diabet/preventive

\begin{tabular}{c|c|c|c|c|}
\hline $\begin{array}{c}\text { Series } \\
\text { Tests }\end{array}$ & $\begin{array}{c}\text { 1.Control } \\
\text { series } \\
\text { (Placebo) }\end{array}$ & $\begin{array}{c}\text { 2.Streptozotoci } \\
n \text { diabetes }\end{array}$ & $\begin{array}{c}\text { 3.Preventive } \\
\text { introduction } \\
\text { of EATM }\end{array}$ & $\begin{array}{c}\text { 4.Therapeutic } \\
\text { introduction } \\
\text { of EATM }\end{array}$ \\
\hline $\begin{array}{l}\text { Number } \\
\text { of } \beta \text {-cells }\end{array}$ & $90,2 \pm 19,9$ & $22,5 \pm 11,2$ & $27,0 \pm 9,5$ & $39,2 \pm 14,1$ \\
\hline
\end{tabular}

Table 2. Effect of the EATM on the number of pancreatic $\beta$-cells in STZ-induced rats pancreas

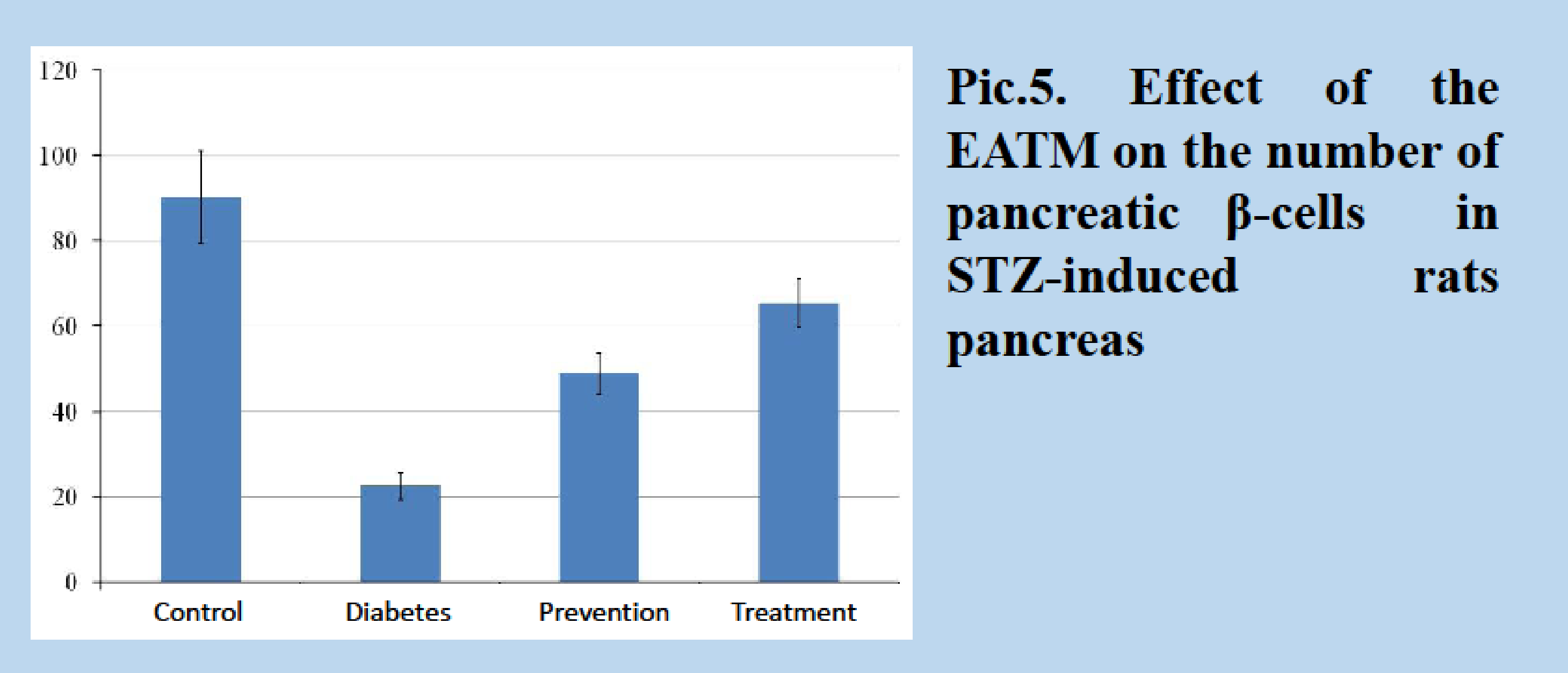

The EATM suppresses Nox releasing (pic.6) from erythrocyte membranes (EM) of blood samples drawn from STZ induced rats and those of control rats blood by $45.5 \pm 6.2 \%(p=0.001 ; n=12)$. It was found that under the specified conditions suppression of EATM-induced Nox release process in EM occurred in a dose dependent manner (pic.7). EATM suppresses Nox release from different membrane structures and restores the mechanism of insulin signal transduction, thus maintaining the euglycemia. An increase in Nox cleavage with increasing lipid peroxidation adequately reduces stability of these membranes, which is a novel mechanism of biological membranes destabilization. Suppression of the Nox isoforms releasing process, both from EM and exosomes in STZ induced rats under the influence of EATM is a novel mechanism of its membrane-stabilizing effect ex vivo. This sets the ground for possible therapeutic application of EATM for stabilizing the EM and exosomes in patients with diabetes.

Conclusion: In rats with STZ-induced diabetes both preventive and therapeutic mode of EATM injection revealed definite protective effect. The normalization of studied biochemical, immunological and morphological indices was observed.
Pic.6. Optical absorption spectra of Nox released from EM obtained from blood of control and STZ induced diabetes rats after 4 days of aerobic incubation of aqueous mixtures at $4^{\circ} \mathrm{C}$ and $\mathrm{pH} 8$ in the absence (solid lines) and presence (dashed lines) of $0.15 \mathrm{mg} / \mathrm{ml}$ of EATM. The absorption spectra of Nox: EM of control blood $(1,2)$; EM of blood samples drawn from STZ rats $(3,4,5,6)$ rats. Maximum optical absorptions for Nox at 560, 530 and $412 \mathrm{~nm}$ (oxidized) are shown to be similar to the rest of the Nox spectra.

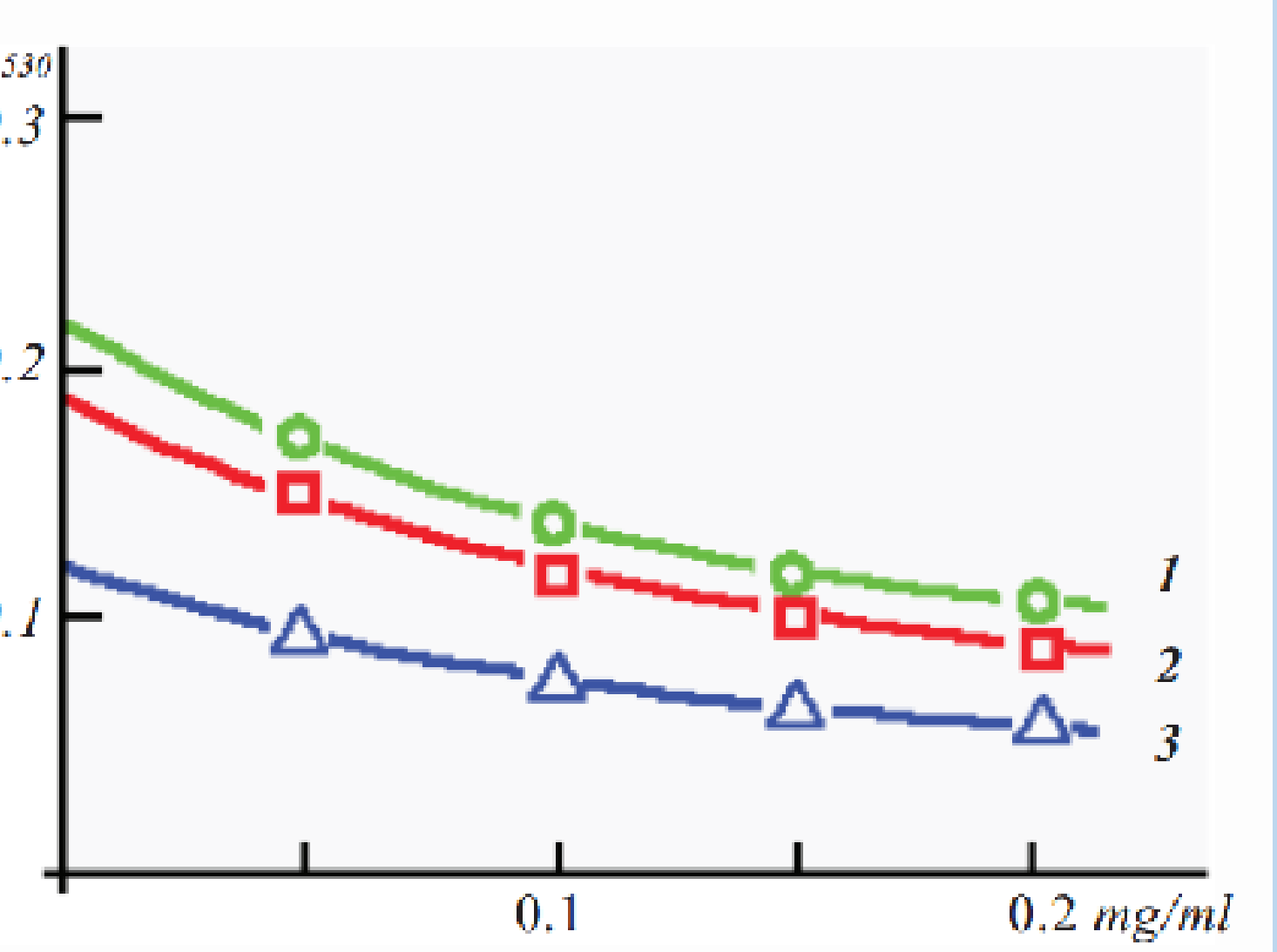

Pic.7. Changes in density of maximum optical absorption of Nox (B-absorption band) from the blood of STZ indused and control rats at $530 \mathrm{~nm}$ under the influence of EATM various doses. The density of EM Nox optical absorption in STZ induced $(1,2)$, and control rats blood (3) is demonstrated. 